\title{
From Bench to Clinical Using a Combinatorial Screenings of Phage-Displayed Peptide Libraries
}

\author{
Nelson Santiago Vispo ${ }^{1}$ and Hortensia Rodríguez Cabrera ${ }^{2}$ \\ ${ }^{I}$ Prometeo Researcher SENESCYT, School of Biology, Yachay Tech, Yachay; ${ }^{2}$ School of Chemistry, \\ Yachay Tech, Yachay City of Knowledge, Urcuqui, Ecuador
}

\section{Introduction}

\section{Phage peptide display technology}

Phage peptide display technology was not initially designed to develop cyclic peptide compounds when it was first introduced [1]. In this technology, each phage particle displays a unique peptide on its surface and the winner can be selected for binding toward a target molecule. Usually, peptides are displayed on the $N$-terminus, the middle, or the $C$-terminus of coat proteins, and the peptide sequence from each phage particle is directed by the DNA sequence of the same phage particle, allowing an easy sequence determination. The selection can be repeated as long as encoding DNA molecules are preserved, and this repeated procedure, called biopanning, is used to enrich the best binders. Billions of pooled peptides presented on phage particles form a phage displayed peptide library, and in contrast to regular synthetic small molecule libraries, as many as $10^{10}$ different peptides can be screened simultaneously for the desired activity [2].

\section{Cyclic Peptides}

Cyclic peptides are polypeptide chains taking ring structures, typically bind with much higher affinity to target proteins than linear ones, due to a smaller reduction in entropy upon binding, allowing an enhanced binding toward target molecules, or better receptor selectivity $[3,4]$. The ring structure can be formed by linking one end of the peptide and the other with chemically stable bonds Head-to-tail cyclization is the amide bond formation between amino and carboxyl termini, and many biologically active cyclic peptides are formed by this way. However, cyclic peptide libraries are most easily generated by forming a disulfide bridge between two cysteine's within the peptide [5] (Figure 2).

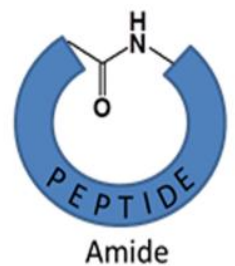

Amide

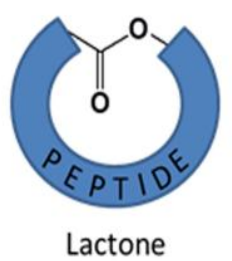

Lactone

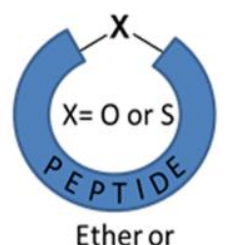

thioether

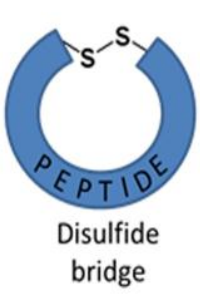

Fig. 2. Chemically stable bonds used to cyclize linear peptide sequences.

\section{Additional advantage of the cyclic peptides:}

- Higher proteolytic stability, due to the lack of both, amino and carboxyl termini. Cyclic peptides can be resistant even to endopeptidases.

- Structures less flexible (more rigid) than linear peptides, particularly important aspects for its therapeutic applications.

In order to increase the flexibility of phage display technology, it is desirable to be able to impose some structural constraints on the peptides that are presented by the phage particles. Cyclized peptides from disulfide bridges were sometimes obtained from phage display, for instance the RGD peptide, and a cyclic peptide can be prepared by designing the peptide sequences such as Cys-X6-Cys for 
platelet glycoprotein binding [6]. The phage particles are released to oxygen rich periplasmic space of bacteria, and two neighboring Cys residues would naturally form a disulfide bond to yield a cyclic peptide [7].

Using a peptide-based approach and exploiting the feasibility of cell permeable peptides as carriers, we describe the identification and characterization of proapoptotic cyclic peptide that impairs the phosphorylation by CK2 in vitro and that leads to regression when administered directly to solid tumors. This peptide, called CIGB 300, was originally identified by screening a 9-mer random linear and cyclic peptide phage library and by using the human papillomavirus (HPV-16) E7 acidic domain as target. The cyclic peptide obtained compared to the linear peptide selection show an increased biological activity [8]. CIGB-300 elicits antitumor activity in different animal models when administrated by different routes [9]. As an investigational drug, CIGB-300 was previously tested in a First-in-Human trial where patients with high grade squamous intraepithelial lesion (HSIL) or epidermoid microinvasive cervical cancer received intralesional injections of this peptide-based drug [10]. That was the first clinical trial in which a drug was used to target the CK2 phosphoacceptor domain providing an early proof-of-principle of a possible clinical benefit. Treatment with CIGB-300 significantly reduced the B23/nucleophosmin levels in tumor specimens. CIGB-300 meets potentialities to be tested in future trials in a neoadjuvant setting prior to chemoradiotherapy in cervical cancer [11] (Figure 2).

\begin{tabular}{cc}
\hline $\begin{array}{c}\text { Phage } \\
\text { clones }\end{array}$ & $\begin{array}{c}\text { 9-mer cyclic peptide } \\
\text { phage library obtained } \\
\text { after biopanning }\end{array}$ \\
\hline 1 & CRNCTVIQFSC \\
4 & CFQPLTPLCRC \\
5 & CQSYHELLLQC \\
6 & CQIPQRTATRC \\
10 & CSVRQGPVQKC \\
12 & CSSCQRTDPGYC \\
14 & CAKQRTDPGYC \\
CIGB 300 & CWMSPRHLGTC \\
18 & CHYIAGTVQGC \\
22 & CPLVSLRDHSC \\
24 & CKQSYLHHLLC \\
\hline
\end{tabular}
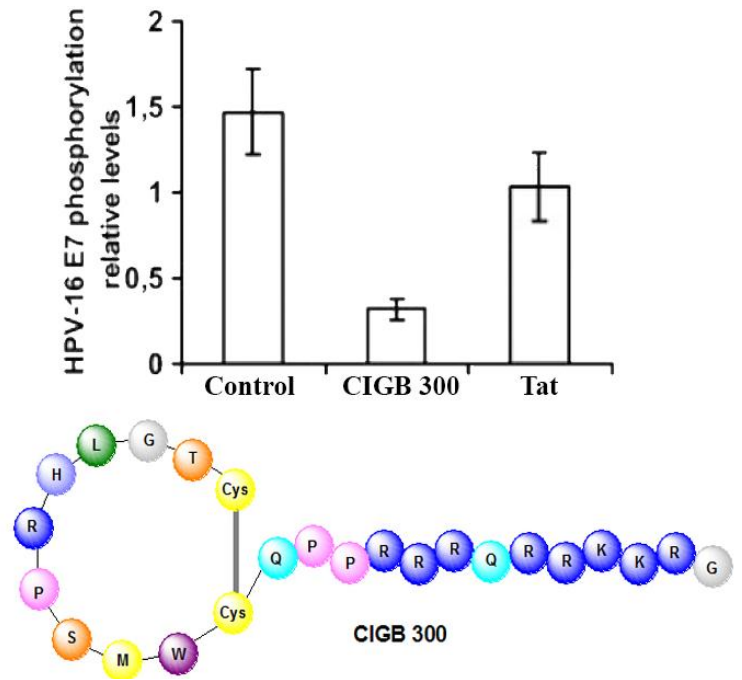

Fig. 2. Structures of cyclic peptides from the cyclic peptide phage display libraries. Phosphorylation was done with a blue stained gel Levels of the 32P-HPV16 E7-GST protein were normalized with respect to levels of the Coomassie-stained HPV-16 E7-GST. CIGB 300 cyclic peptide with penetration peptide.

\section{Peptide Synthesis}

After the selection and identification (sequentiation) of targeted peptides through phage displayed, the sequences have to be synthesized. Taking to account that in general, the selected peptides by this methodology have short and more or less simple sequences, in the majority of reported cases, the solid phase peptide synthesis (SPPS) is the method of choice.

From Merrifield (1963) until today, the solid-phase principle for peptide synthesis has been conceived and developed with the purpose of providing a rapid, simplified, and effective way to prepare peptides and small proteins. In this sense, Fmoc/t-Bu method, which is based on an orthogonal protecting group strategy, uses the base-labile N-Fmoc group for protection of the $\alpha$-amino group and acid-labile side-chain protecting groups and resin linkage agents. 
Since removal of temporary and permanent protection is effected by completely different chemical mechanisms, side-chain protecting groups and linkage agents can be employed, and are removed under considerably milder conditions than those used in the Merrifield method.

With the linear peptide in hand, the cyclization step becomes the main challenge. Certainly, this procedure, specifically through disulfide bond formation, is one of the most important step in the preparation of these compounds and many successfully strategies have been reported. It also should be mentioned that some selected cyclic sequences have been obtained in the biotinylated form [12].

\section{Cyclization Particularities (Examples)}

CIGB-300: CIGB-300 was synthesized on solid phase (Fmoc/t-Bu strategy), and an additional cyclization step was done by oxidation of the linear peptide in ammonia aqueous solution $(\mathrm{pH} 8.2)$ for 72 hours with agitation [13].

Cyclic Peptide Ligands for NeutrAvidin and Avidin: The selected NeutrAvidin-binding peptides were also synthesized on solid phase (Fmoc/t-Bu strategy), using RinkAmide-AM resin as solid support. Peptide cyclization was carried out by oxidation of the two cysteines to form an intramolecular disulfide bond. The peptides were shaken in phosphate-buffered saline (PBS; pH 7.4) with 10\% dimethyl sulfoxide (DMSO) for $8 \mathrm{~h}$ at $37^{\circ} \mathrm{C}$. Extent of the disulfide bond formation was monitored as a loss of free thiol using Ellman's reagent.

Others examples of the successful use of this approach are the identification of ligands of tumor necrosis factor- $\alpha$ (TNF- $\alpha)$ [14], neutravidin and avidin, and inhibitors of LEDGF/p75PPI that prevent HIV replication [15].

One drawback of phage display is that the cyclic peptides are formed by disulfide formation, not head-to-tail cyclization. In addition, phage display is limited to natural, ribosomal amino acids. Many amino acids found in biologically active cyclic peptides are non-ribosomal, and they are not accessible with phage display.

\section{Conclusions}

Cyclic peptides show better biological activity compared to their linear counterparts due to its conformational rigidity. Biologically active cyclic peptides have been identified through phage display technology and both, solid phase synthetic approaches and cyclizations strategies, have allowed to obtain this potentially biologically active structures for further studies, showing their usefulness as therapeutics and biochemical tools. Cyclic peptides have several structural features making them good drug leads, and there are several naturally occurring cyclic peptides in clinical use.

\section{Acknowledgments}

This work was sponsored by the Prometeo Project of the Secretariat for Higher Education, Science, Technology and Innovation of the Republic of Ecuador.

\section{References}

1. Smith, G.P. Science 228, 1315-1317 (1985), http://dx.doi.org/10.1126/science.4001944

2. Azzazy, H.M., et al. Clin. Biochem. 35, 425-445 (2002), http://dx.doi.org/10.1016/S0009-9120(02)00343-0

3. Edman, P.E.H.R. Ann. Rev. Biochem. 28, 69-96 (1959), http://dx.doi.org/10.1146/annurev.bi.28.070159.000441

4. Horton, D.A., et al. J. Comp. Aided Mol. Des. 16, 415-431 (2002), http://dx.doi.org/10.1023/A:1020863921840

5. Wilkins, M.R., et al. The Lancet 349, 1307-1310 (1997), http://dx.doi.org/10.1016/s0140-6736(96)07424-7

6. O'Neil, K.T., et al. Struct. Funct. Bioinform. 14, 509-515 (1992), http://dx.doi.org/10.1002/prot.340140411

7. Joo, S.H. Biomolecules \& Therapeutics 20, 19-26 (2012), http://dx.doi.org/10.4062/biomolther.2012.20.1.019

8. Perea, S.E., et al. Cancer Res. 64, 7127-7129 (2004), http://dx.doi.org/10.1158/0008-5472.CAN-04-2086

9. Perera, Y., et al. Inter. J. Cancer 122, 57-62 (2008), http:///x.doi.org/10.1002/ijc.23013

10. Solares, A.M., et al. BMC Cancer 9, 146 (2009), http://dx.doi.org/10.1186/1471-2407-9-146

11. Soriano-García, J.L., et al. J. Cancer Res. Ther. 1, 153-162 (2013), http://dx.doi.org/10.14312/2052-4994.2013-25

12. Sclavons, C., et al. Inter. J. Pept. 2013, 9 (2013), http://dx.doi.org/10.1155/2013/348409

13. Cabrales-Rico, A., et al. J. Pharm. Biomedical Analysis 105, 107-114 (2015), http://dx.doi.org/10.1016/j.jpba.2014.11.043

14. Meyer, S.C., et al. Chem. Biol. Drug Des. 68, 3-10 (2006), http://dx.doi.org/10.1111/j.1747-0285.2006.00401.x

15. Desimmie, B.A., et al. Mol. Ther. 20, 2064-2075 (2012), http://dx.doi.org/10.1038/mt.2012.132 